\title{
Multiband photometry towards the inner Galactic bulge: extinction, atmospheric parameters, and metallicities ${ }^{\star, \star \star, \star \star \star}$
}

\author{
B. X. Santiago ${ }^{1}$, S. C. Javiel ${ }^{1}$, and G. F. Porto de $\mathrm{Mello}^{2}$ \\ 1 Universidade Federal do Rio Grande do Sul, IF, CP 15051, Porto Alegre 91501-970, RS, Brazil \\ e-mail: santiago@if.ufrgs.br; [basilio.santiagos; sandro.javiel]@ufrgs.br \\ 2 Observatório do Valongo, Universidade Federal do Rio de Janeiro, Ladeira do Pedro Antonio 43, \\ 20080-090 Rio de Janeiro, Brazil \\ e-mail: gustavo@ov.ufrj.br
}

Received 12 January 2006 / Accepted 3 May 2006

ABSTRACT

\begin{abstract}
Context. We have obtained BVRI photometry for 21 stellar fields located in directions towards the central $3.5^{\circ}$ of the Galaxy. Each field is $10 \times 10$ arcmin in size, and a total of $\sim 2 \times 10^{5}$ stars were measured.

Aims. Previous analyses based on 2 Micron All Sky Survey (2MASS) data have shown that these directions have a relatively low extinction $\left(A_{K} \simeq 0.25-0.30\right)$. The combined optical-infrared sample contains about $3.5 \times 10^{4}$ stars. This large dataset provides a more detailed extinction map within these surveyed areas than previously derived. The large number of colour indexes available also yielded estimates of effective temperatures and metallicities for the inner bulge stars.

Methods. Extinction and effective temperature were estimated for each star based on comparing the observed colours to model predictions. The extinction-corrected colour magnitude diagrams indicate the presence of a broad red giant branch of inner bulge stars detached from the helium-burning red clump of both bulge and disc stars. The red giant stars belonging to the bulge form a wide sequence mostly due to variable metallicity. As previously done by other authors, we derived photometric metallicities by comparing the position of each bulge red giant to a set of templates based on Galactic globular clusters.

Results. We confirm the existence of regions with $A_{K}<0.30$ in these fields, as attested by analysis of the stellar colours. The resulting metallicity distribution function is peaked at $[\mathrm{Fe} / \mathrm{H}] \simeq-0.25$ and extends to supersolar values up to $[\mathrm{Fe} / \mathrm{H}] \simeq 0.3$. This distribution is similar among the 4 regions, with relatively small field-to-field variations within the central $1 \mathrm{kpc}$. Our results are also in good agreement with the metallicity distributions from other authors, both photometric and spectroscopic. Put together, this result strongly limits the existence of an abundance gradient in the inner bulge.
\end{abstract}

Key words. Galaxy: abundances - Galaxy: bulge - Galaxy: structure - Galaxy: stellar content - Galaxy: center - dust, extinction

\section{Introduction}

The central region of the Galactic bulge is still relatively unstudied due to the high extinction values in the optical, the high surface density of stars, and the contaminating effects of the foreground disc structure. Most of what is known about the stellar content of the Galactic bulge is based on relatively external regions more than 6 degrees from the Galactic centre, or on more central windows where extinction was found to be lower than average, such as Baade's window (Baade 1951; hereafter BW). But even this latter, on which a large amount of work has been done, is located at $4^{\circ}$ from the centre of the Galaxy.

Closer than $\simeq 4^{\circ}$ from the Galactic centre, most of the work is recent and based on near infra-red (NIR) surveys. Catchpole et al. (1990) studied the distribution of stars within the central $2^{\circ}$ and derived high extinction values, $A_{V}>7$. Frogel et al. (1999) determined extinction and metallicities for 11 fields close to the

* Based on observations collected at the Observatório do Pico dos Dias, operated by the Laboratório Nacional de Astrofísica, CNPq. Brazil.

$\star \star$ Tables for $B V R I J H K$ photometry and positions are only available in electronic form at the CDS via anonymous ftp to cdsarc.u-strasbg.fr $(130.79 .128 .5)$ or via

http://cdsweb.u-strasbg.fr/cgi-bin/qcat? J/A+A/458/113

$\star \star \star$ Appendix $\mathrm{A}$ is only available in electronic form at

http://www. aanda.org minor axis of the Galaxy. Their derived average $A_{K}$ values for these fields are in the range $0.27<A_{K}<2.15\left(2.4 \leq A_{V} \leq 19\right)$. The advent of wide-angle NIR surveys, such as the Deep NIR Southern Sky Survey (DENIS, Epchtein et al. 1997) and the Two Micron All Sky Survey (2MASS, Skrutskie et al. 1997), provides an enormous amount of data on the central bulge. Schultheis et al. (1999) used DENIS data and found extinction values from $A_{V} \simeq 6$ up to $A_{V} \simeq 37$ in the central regions along the disc plane $\left(|b| \leq 1.5^{\circ}\right)$. Based on 2MASS data, Dutra et al. (2003) present an extinction map of the central $5 \mathrm{deg}$ of the Galaxy. Dutra et al. (2002) studied two regions of about 30 arcmin in size and with $A_{K} \simeq 0.28$.

These inner regions of relatively low extinction present new opportunities for detailed studies of the stellar populations at different positions along the Galactic bulge. In particular, stellar metallicities and abundance ratios provide strong constraints to scenarios for the formation of the bulge. Similar to other bulge properties, most of the work concerning stellar metallicities has so far been carried out farther than the central $4^{\circ}$ or at BW. Minitti et al. (1995) obtained mid-resolution spectra of several hundred stars beyond $1.5 \mathrm{kpc}$ from the Galactic centre and discussed evidence in favour of a metallicity gradient in the Galactic bulge within $2 \mathrm{kpc}$ from the centre. Combining photometric and spectroscopic data, Ibata \& Gilmore (1995) studied several fields in outer-Bulge regions, deriving metallicity distribution functions (MDF) for each field. They found no evidence 
of a metallicity gradient in the outer Bulge. Sadler et al. (1996) and McWilliam \& Rich (1994) obtained metallicities for giant stars in BW and also built MDF from the data. These MDFs are broad and peak at $[\mathrm{Fe} / \mathrm{H}] \simeq 0$ and $[\mathrm{Fe} / \mathrm{H}] \simeq-0.2$. Ramirez et al. (2000) spectroscopically analysed some inner bulge regions along the minor and major axes and derived an MDF that is consistent with those of previous authors. Their results are consistent with no metallicity gradient in the central $0.5 \mathrm{kpc}$.

Photometric metallicity analyses of bulge stars have also been carried out. Tiede et al. (1995) used NIR colour-magnitude diagrams to obtain average metallicities in different fields along the bulge minor axis, resulting in a gradient $\mathrm{d}[\mathrm{Fe} / \mathrm{H}] / \mathrm{d} b=$ $-0.060 \pm 0.033 \mathrm{dex} \mathrm{deg}^{-1}$ within $-12^{\circ} \leq b \leq-3^{\circ}$. Frogel et al. (1999) extended this analysis towards inner regions, along the same minor axis, and obtained similar results. More recently, Zoccali et al. (2003) combined optical and NIR data to analyse a region at $6^{\circ}$ from the centre and obtained a MDF that peaks at about the same value as those from spectroscopy but with a faster drop at the high metallicity end.

The Galactic bulge is also known to be undergoing substantial star formation within its central molecular zone, with young stellar populations being found within inner few hundred parsecs (Serabyn \& Morris 1996; Morris \& Serabyn 1996). The bulge windows studied here lie in a transition region between the nuclear and the older, more extended bulge components (Launhardt et al. 2002), which are therefore of interest in constraining scenarios for the formation and heating of young stars due to bulge dynamics (Kim \& Morris 2001).

In this paper we present optical photometry for 4 fields which, according to the extinction maps by Dutra et al. (2003), are suspected of having much lower than average extinction in the central $3.5^{\circ}$ from the Galactic centre. Two of them were identified by Dutra et al. (2002); the other two are relatively unstudied extensions of the BW and SgrI window, which remain of low extinction according to the 2MASS maps. The resulting optical and optical-infrared colours are compared to model predictions, resulting in extinction values and atmospheric parameters, such as effective temperatures and metallicities. This paper is organised as follows: Sect. 2 presents the optical data, Sect. 3 discusses the tools used in the data analysis. In Sect. 4 we present the results in terms of extinction, effective temperatures $\left(T_{\text {eff }}\right)$ and photometric metallicities for the stars in the sample. Finally, we present our discussion and conclusions in Sect. 5.

\section{The data}

The optical observations were carried out with the $0.6 \mathrm{~m}$ telescope at Pico dos Dias, Laboratório Nacional de Astrofísica (LNA), Brazil, on the nights of 26 and 27 June 2003. The nights were photometric with a typical seeing varying from 1 to 1.5 arcsec (in $B$ band). A SITe CCD with $1024 \times 1024$ pixels was used. It provides a $0.61^{\prime \prime} /$ pixel scale and has a quantum efficiency of $>50 \%$ within the optical bands used. A total of 21 fields, of $10 \times 10$ arcmin each, were observed with $B V R I$ filters. These fields were located in the 4 low extinction regions, defined previously by using the 2MASS extinction maps of Dutra et al. (2003). The window name, its central galactic coordinates, the number of LNA fields covered in each region, the total exposure time in each band per field, and total number of stars measured are listed in Table 1. The exposure times were enough to reach an $S / N \simeq 10$ for $V \sim 18$ stars, which corresponds to the typical magnitude of evolved red giants at the distance to the Galactic centre.
Table 1. Bulge and clusters fields.

\begin{tabular}{lcccccccc}
\hline \hline Field name & $\begin{array}{c}l \\
(\mathrm{deg})\end{array}$ & $\begin{array}{c}b \\
(\mathrm{deg})\end{array}$ & $N_{\text {flds }}$ & $\begin{array}{c}t_{B} \\
(\mathrm{~s})\end{array}$ & $\begin{array}{c}t_{V} \\
(\mathrm{~s})\end{array}$ & $\begin{array}{c}t_{R} \\
(\mathrm{~s})\end{array}$ & $\begin{array}{c}t_{I} \\
(\mathrm{~s})\end{array}$ & $N_{\text {stars }}$ \\
\hline W0.8-3.3 & 0.80 & -3.30 & 10 & 240 & 120 & 90 & 90 & $1.50 \times 10^{5}$ \\
W1.5-2.55 & 1.50 & -2.55 & 9 & 240 & 120 & 90 & 90 & $1.35 \times 10^{5}$ \\
W0.2-2.2 & 0.25 & -2.15 & 1 & 240 & 120 & 90 & 90 & $1.20 \times 10^{4}$ \\
W359.5-3.0 & 359.4 & -3.1 & 1 & 240 & 120 & 90 & 90 & $1.05 \times 10^{4}$ \\
NGC 3960 & 294.4 & 6. & 1 & 80 & 80 & 70 & 47 & 1339 \\
IC 4651 & 340.1 & -7.9 & 3 & 60 & 16 & 8 & 8 & 1664 \\
\hline
\end{tabular}

Notice that some of these windows have been identified earlier by other authors, as possible directions of relatively low extinction. W0.2-2.2 was first identified by Stanek (1998) using DIRBE/IRAS but has not been studied in detail. W0.8-3.3 is located not far from BW, being $\simeq 0.7^{\circ}$ closer to the galactic centre. W1.5-2.55 is essentially an extension of the Sgr I window, which was originally also identified by Baade at coordinates $l=1.25^{\circ}$ and $b=-2.65^{\circ}$.

Two old open star clusters with available optical colourmagnitude diagrams and well known reddening, distance moduli, and metallicity were also observed on the same nights. They were used as a cross-check of the photometric calibration. Their location and exposure times are also listed in Table 1.

Instrumental magnitudes were obtained from point-spreadfunction (PSF) fitting photometry. Tests were run to check for the sensitivity of the PSF with time during the night or chip location. The PSF proved to be quite stable. Therefore, model PSFs were built for each filter and for each observation night using bright but non-saturated photometric standard stars. Different stars on different exposures were combined on a PSF template from which the model PSF was built. The input magnitudes to the PSF fitting process were aperture magnitudes in a 2 pix $\left(=1.22^{\prime \prime}\right)$ radius aperture for all stars and bands. This choice of a small aperture was necessary due the field crowding. Aperture corrections, derived from the bright isolated standard stars, proved to be stable and therefore were applied during the process of calibration to the standard system.

\subsection{Photometric calibration and astrometric solutions}

Several different photometric standard stars from the list of Landold (1992) were observed during the two nights. These standards were chosen so as to have different colours and were observed at various airmasses to provide adequate calibration transformations. A total of $6 \mathrm{SA}$ fields, containing more than one star within the LNA telescope field of view were repeatedly observed during the run. Furthermore, two open clusters, NGC 3960 and IC 4651, were observed during the run in order to test the calibration and the photometry in general. Both are old clusters with developed giant branches and available CCD data in the literature (NGC 3960: Prisinzano et al. 2004; IC 4651: Anthony-Twarog et al. 1988; Piatti et al. 1998; and Kjeldsen \& Frandsen 1991).

Figure 1 shows the residuals of the calibration process as a function of the standard star magnitudes. It is clear from the figure that there is no bias in the calibration process. The observed Bulge fields are mutually overlapping to allow repeated photometric measurements of a fraction of the stars, which in turn yields reliable photometric uncertainties (see Sect. 2.2 below). In order to match the stars from the different fields and find coincidences, chip $(x, y)$ positions were converted into equatorial coordinates with a multi-step process. 


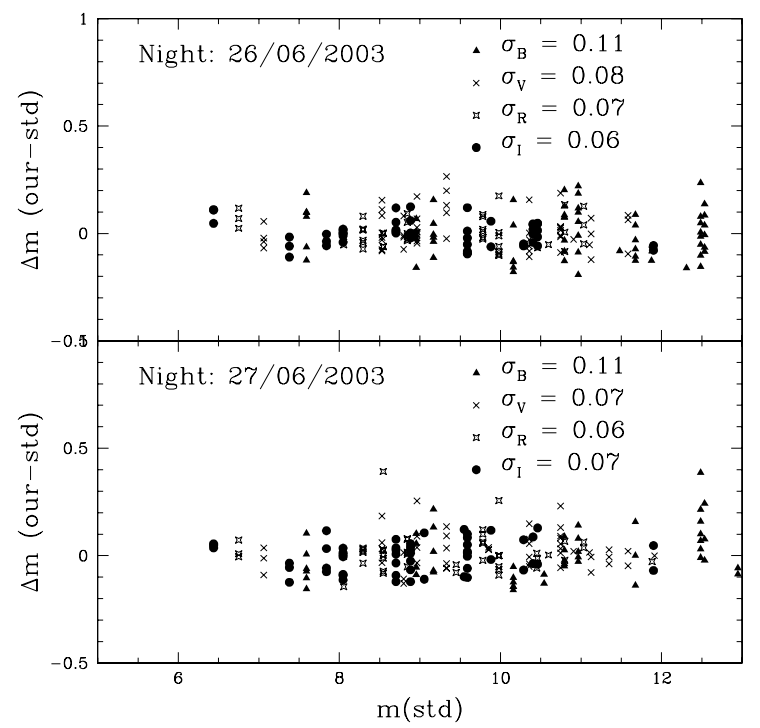

Fig. 1. Panel a) Calibration residuals for the night of June 26, 2003. The residuals are shown against the standard magnitudes. The different symbols correspond to the different filters, as indicated. Panel b) The same as in panel a, but now showing the calibration residuals for the night of June 27.

In the online Appendix A, we give details of the observations of calibration standards and clusters and describe the photometric calibration procedure. We also present the astrometric method used to convert chip coordinates into equatorial ones. Eletronic tables, one per bulge window, are also provided at the CDS. They contain the following information for each star: Col. 1: right ascension (J2000); Col. 12: declination (J2000); Col. 3: $B$ magnitude; Col. 4: $B$ error; Col. 5: $V$ magnitude; Col. 6: $V$ error; Col. 7: $R$ magnitude; Col. 8: $R$ error; Col. 9: $I$ magnitude; Col. 10: I error; Col. 11: 2MASS $J$ magnitude; Col. 12: 2MASS $H$ magnitude; Col. 13: 2MASS $K$ magnitude.

\subsection{Photometric uncertainties}

Most of the 21 fields imaged were mutually overlapping. This allowed us to obtain repeated magnitude measurements for about one third of the stars in the sample. Given the stable astrometric transformation from $\mathrm{CCD}$ coordinates, we ran a positional matching algorithm that looked for individual stars with photometric measurements in a given field and that were the closest positional match to a given star in another field. They were considered to be independent measurements of the same star if, besides being the closest match, their distance on the sky was $1^{\prime \prime}$ or less and if their magnitude difference was smaller than $1 \mathrm{mag}$.

About $1 / 3$ of the stars in the sample had at least two independent magnitude measurements in the four filters, according to these criteria. For this subsample, photometric errors were computed as the dispersion of the individual measurements relative to the mean value. The uncertainties calculated in this way are shown as a function of the mean magnitudes in Fig. 2. The uncertainties clearly increase at fainter magnitudes, as expected. The upper border in the error distribution reflects our cut-off criterion in magnitude difference for a valid match. However, the number of stars that reach this artificial upper limit is small. And many of these cases are mismatched stars in our crowded fields. The bulk of the stars have much smaller photometric errors: $80 \%$ of the stars with $V \leq 18$ have errors smaller than $\Delta V=0.10$. We therefore use this as the cut-off magnitude limit to our sample.
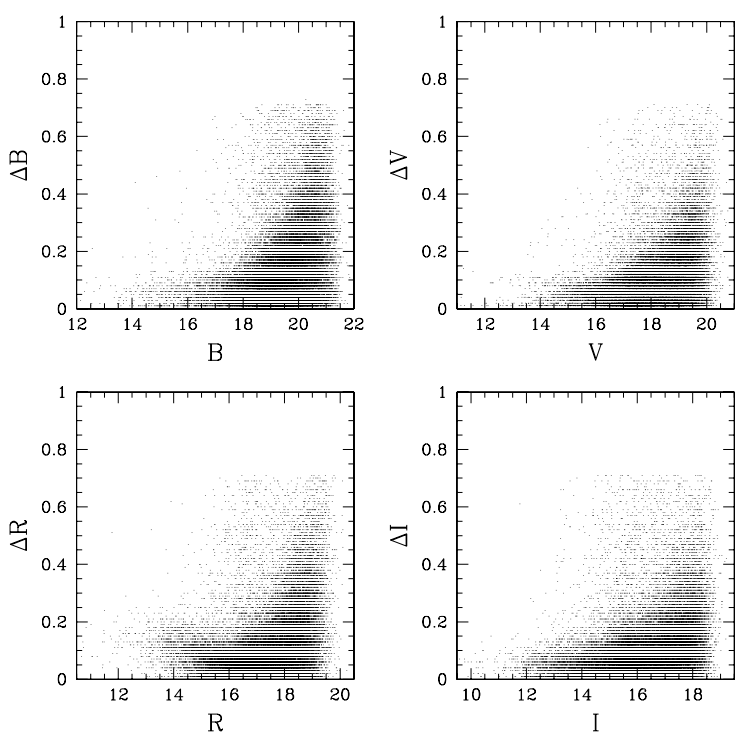

Fig. 2. Magnitude errors for the subset of stars in the window W1.52.55 , for which repeated photometric measurements were made. The errors are shown as a function of the average magnitude for each star, which was considered to be the final measurement. The different panels correspond to different filters, as indicated.

\section{Data analysis}

Even though the fields are close to the direction towards the central bulge, the Galactic disc is certainly a contaminating foreground component whose contribution to the star sample must be considered. Given the low galactic latitudes and the direction towards the centre, the thin disc will by far dominate the counts relative to the thick disc or the outer spheroid (halo). We computed the predicted disc star number counts by assuming that the disc is described by a exponential density profile along the disc plane with a scale length of $2.5 \mathrm{kpc}$. The model also assumes an exponential vertical profile with a scale height of $250 \mathrm{pc}$, and assumes that the stars follow a Wielen luminosity function. The absorbing dust follows a Galactic extinction law, and the dust density profile is taken to be uniform along the disc plane and to follow an exponential with $h_{\text {dust }}=100$ pc scale height perpendicular to it.

The model star number counts as a function of magnitude are shown for a typical field in the W1.5-2.55 window in Fig. 3 for different choices of the total line of sight extinction coefficients $A_{V}$. The actual $V$ magnitude counts are shown in the figure for comparison. Notice that even for $A_{V}=1.5$ the predicted counts are still smaller than the observed ones in most magnitude bins. This corresponds to $A_{K}<0.17$, which is a significantly lower value than typically found by Dutra et al. (2002). Therefore, assuming a more typical $A_{V}=2$, we conclude that our fields do sample the central bulge, although with a significant contamination by foreground disc stars.

This conclusion is strengthened if we use a holed disc model as suggested by several authors (Lépine \& Leroy 2000; Picaud \& Robin 2006). Figure 3 shows the corresponding number counts based on the model by Picaud \& Robin (2006). The expected counts are actually larger for $V \lesssim 14.5-15.0$ but flatten out considerably beyond that, whereas the observed counts keep rising. Similar results apply to the other fields and windows under investigation, in the sense that in all of them there is a clear excess of observed stars relative to pure disc models. 


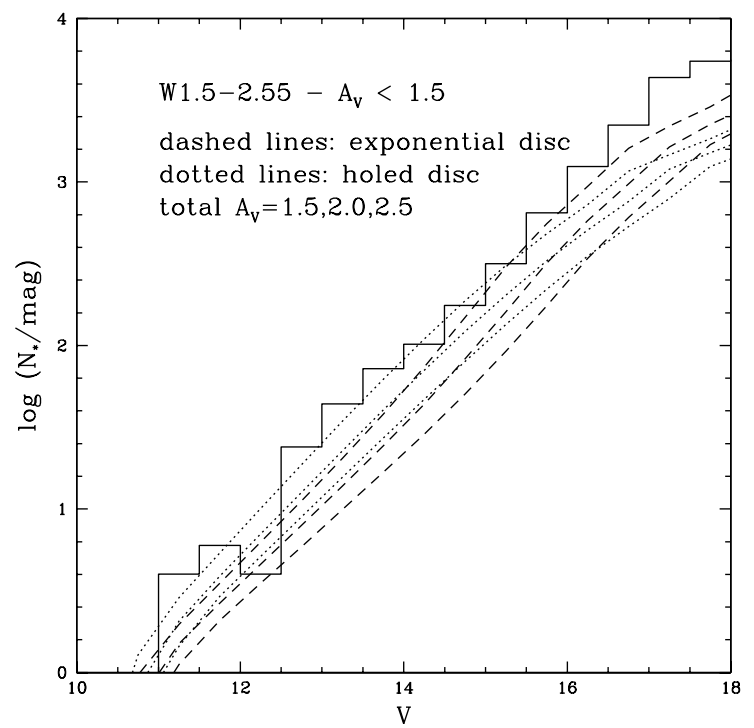

Fig. 3. Star number counts per unit magnitude on a logarithmic scale. The histogram is the observed number count for our LNA sample towards the field w1.5-2.55_3_5e. The dotted lines are the expected number counts based on an exponential disc model for different values of extinction at infinity. From top to bottom: $A_{\mathrm{V}}=1.5, A_{\mathrm{V}}=2.0$, and $A_{\mathrm{V}}=2.5$.

The disc and bulge populations may also be separated with the help of a colour magnitude diagram (CMD). We chose the $I$ vs. $(V-I) \mathrm{CMD}$ as an example. The CMDs of all 4 windows are shown in Fig. 4. The two first windows were covered by several LNA fields, so their CMDs contain a much larger number of stars. Only stars with $V<18$ mag are shown. This magnitude cut-off is responsible for the diagonal limiting line at the bottom of the CMDs. Colour error bars are shown in the lower left panel. They were estimated from Fig. 2 by adding the rms $I$ and $V$ magnitude errors for $(V-I)=2.0$ stars in quadrature. These colour errors are representative of all the data. In all 4 panels we distinctly see 3 loci standing out at brighter magnitudes: the one on the left $((V-I) \simeq 1.0)$ is the disc main sequence, containing stars of $\mathrm{F}$ and $\mathrm{G}$ types; at $(V-I) \simeq 1.6$ and $I<14$ one sees disc stars belonging to the red clump (RC) spread out vertically due to their variable distances. This sparser locus is barely visible in the W0.2-2.2 and W359.5-3.0 windows; and finally, a large cloud stretching redwards of $(V-I) \simeq 1.8$ is made up of bulge red giant branch (RGB) and asymptotic red giant branch (AGB) stars.

In order to extract atmospheric parameters and extinction values for each star in the sample, we compared their observed colours to those of model stars. We used two theoretical model grids for this purpose:

1) The models by Bessel et al. (1998, hereafter BCP98). These authors provide optical and infrared colours for a large grid of stars, most of them of solar metallicity. For non-solar abundances, the Bessel et al. models are restricted to relatively cool stars $\left(T_{\text {eff }}<4750 \mathrm{~K}\right)$ and low surface gravities $(\log g<$ 3.5). These models are based on NMARCS model atmospheres by Plez et al. (1992) and Plez (1995).

2) The models by VandenBerg \& Clem (2003, hereafter VC2003). These authors provide only optical colours for two grids: one covering $3000 \mathrm{~K}<T_{\text {eff }}<5500 \mathrm{~K},-0.5<\log g<$ $5.0,-3.0<[\mathrm{Fe} / \mathrm{H}]<0.3$; the other grid covers $4000 \mathrm{~K}<T_{\text {eff }}<$ $40000 \mathrm{~K}, 2.0<\log g<5.0,-3.0<[\mathrm{Fe} / \mathrm{H}]<0.3$.
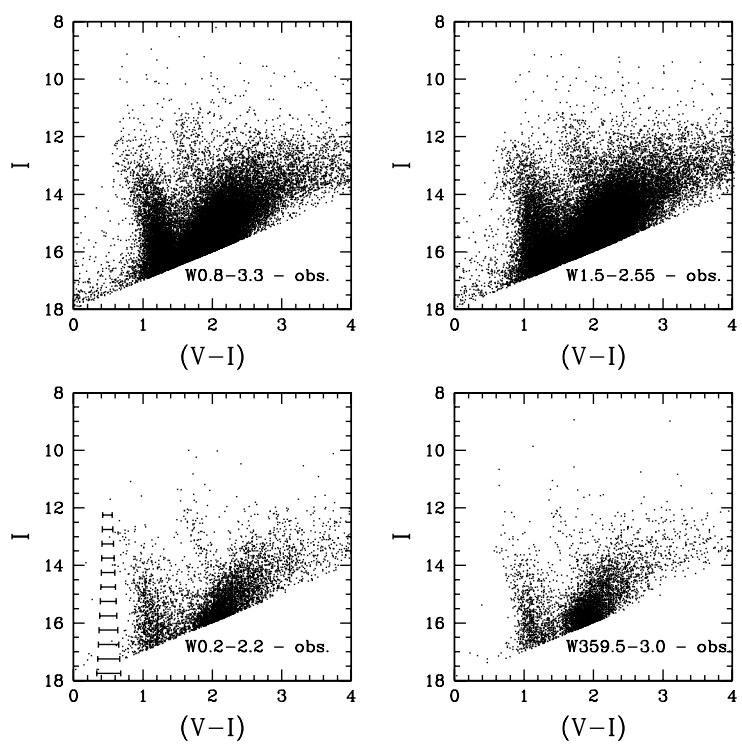

Fig. 4. $I$ vs. $(V-I)$ CMD for each of the 4 bulge regions studied. The window name is indicated in each panel. Colour error bars are shown in the lower left panel for guidance. They were estimated from the error distributions shown in Fig. 2 as explained in the text.

Each model set has its own advantages. The VC2003 models cover a wider range in temperature, metallicity, and surface gravity than the BCP98 models. Besides, the VC2003 models cover a regular grid in the parameter space formed by these variables, whereas the BCP98 models cover a narrower range in $T_{\text {eff }}$ for high log $g$ than for low log $g$ models. On the other hand, BCP98 model colours extend to the near infra-red (NIR), making it easier to carry out a simultaneous fit to atmospheric parameters and extinction.

In fact, our first attempts to use observed vs. model colour comparisons in order to infer $T_{\text {eff }}$ and $A_{K}$ individually for each sample star with $V<18$ was not successful. For the VC2003 models, the availability of only 4 indexes resulted in degenerate solutions and error bars that were too large. With the BCP98 models, systematic biases resulted from the fact that there is a significantly larger number of models with higher $T_{\text {eff }}(>4250 \mathrm{~K})$ than for lower values. In order to achieve stabler results, a single model grid was created by combining VC2003 and BCP98. Since our main goal is to obtain $T_{\text {eff }}$ and $A_{K}$ we averaged the colours of both grids for a fixed $T_{\text {eff }}$, generating a single table of colours vs. temperature. For $T_{\text {eff }}<4750$. we used the BCP98 table and complemented it with the VC2003 table for higher temperatures, up to $T_{\text {eff }}=5500 \mathrm{~K}$. A systematic colour difference of $\simeq 0.04$ exists between the two grids close to the boundary temperature. We therefore changed the VC2003 model colours in order to bring them into agreement with the BCP98 ones, and extrapolated this offset for the higher temperatures covered only by VC2003. Finally, the BCP98 NIR colours were also extrapolated up to $T_{\text {eff }}=5500 \mathrm{~K}$ in order to have NIR coverage. Even though these extrapolations are not the ideal way to build the model grid, for most stars the dominant source of error in our analysis is still the uncertainty in the observed colours. In fact, when this paper was being written, we became aware of a new grid of colour vs. $T_{\text {eff }}$ and $[\mathrm{Fe} / \mathrm{H}]$ relations published by Ramírez \& Meléndez (2005). We compared our mixed model $T_{\text {eff }}$ vs. colour grid with the tables presented by these authors and found that most of the colour differences at a fixed temperature are smaller than 0.04 .

The intrinsic colours of each star were then compared to the model colours and the best values of $T_{\text {eff }}$ and their associated 
uncertainties were obtained. In this comparison, we allowed extinction to vary considerably, between $0.10<A_{K}<0.70$, and found the best $A_{K}$ value simultaneously to the temperature. In order to take advantage of the near infra-red colours available in the BCP98 models, and also keeping in mind that the solutions in $\left(T_{\text {eff }}, A_{K}\right)$ may be degenerate, we restricted our colour fits to those stars for which $2 \mathrm{MASS} J H K_{\mathrm{s}}$ data were also available. This provided a total of 7 independent observed colour terms to be compared to the models. Finally, we applied a cut-off in the quality of the model vs. observed colour fits, as explained in the next section.

\section{Derivation of stellar parameters}

Since we have $B V R I J H K_{\mathrm{s}}$ magnitudes for the stars used in our analysis, we need an objective means to assess which models recover these observed magnitudes best. A model here was considered as a combination of a model star (characterized as a table entry listing its $T_{\text {eff }}$ and its intrinsic colours, as explained in the previous section) and an extinction $A_{K}$ value. We proceeded as follows:

1. For each $A_{K}$ we computed the extinction coefficients in the other filters assuming a Galactic extinction law. The observed magnitudes were then corrected for extinction, and the star's intrinsic colours were computed. The 2MASS $K_{\mathrm{s}}$ magnitudes were converted to $K$ magnitudes before the correction. The adopted conversion was simply $K=K_{\mathrm{s}}+0.04$ for all stars.

2. We then compared the intrinsic colours to each set of model colours. We computed a $\chi^{2}$ as follows:

$\chi_{j}^{2}=\frac{1}{N_{\mathrm{cols}}-1} \frac{\sum_{i}\left(\mathrm{col}_{j, i}-\operatorname{col}_{\mathrm{obs}, i}\right)^{2}}{\sigma_{\mathrm{col}, \mathrm{obs}, i}{ }^{2}}$

where $N_{\text {cols }}=7, \operatorname{col}_{j, i}$ is the $i$ th colour index of the $j$ th model and $\mathrm{col}_{\mathrm{obs}, i}$ is the intrinsic $i$ th colour index for the star, whose photometric uncertainty is $\sigma_{\mathrm{col}, \mathrm{obs}, i}$.

3. We repeated steps 1 and 2 for all $A_{K}$ values and found the minimum $\chi^{2}$ value, $\chi_{\min }^{2}$. We also kept track of all models with $\chi_{\min }^{2} \leq \chi^{2} \leq \chi_{\min }^{2}+\Delta$, where $\Delta$ is a tolerance in the dispersion values based on the typical observed colour uncertainties for a $V<18$ star.

4. For all models within the acceptable range in dispersion relative to the best model as quoted above, we averaged their $T_{\text {eff }}$ and $A_{K}$ values and computed the dispersion in these parameters. The average was taken to be the best solution for the temperature and extinction for the star, with uncertainties given by the dispersion.

This fitting procedure allowed extinction and temperatures to be extracted individually for stars. The uncertainties in these parameters resulting from the algorithm described above are a strong function of the derived $T_{\text {eff }}$ itself. For hotter stars belonging to the RC and lower RGB branch, which make up most of the sample (see Sect. 4.2), the uncertainties are larger: $\delta A_{K} \simeq 0.06$ and $\delta T_{\text {eff }} \simeq 400 \mathrm{~K}$. For the cooler bulge RGB stars, which we use to derive an MDF in Sect. 4.3, the colours are in general more sensitive to temperature and the uncertainties drop dramatically: $\delta A_{K} \simeq 0.03$ and $\delta T_{\text {eff }} \simeq 100 \mathrm{~K}$.

For all subsequent analysis, only stars for which $\chi_{\min }^{2} \leq 10$ are considered. Therefore, our final sample of stars, to be used in the next sections, results from the combination of several selection criteria: i) $V \leq 18$ in order to use stars with magnitude uncertainties $\simeq 0.10$ or less; ii) availability of 4 optical magnitudes
Table 2. Sample size evolution.

\begin{tabular}{lccc}
\hline \hline Region & $V \leq 18$ & $V \leq 18, N_{\text {cols }}=7 V \leq 18, N_{\text {cols }}=7, \chi_{\text {min }}^{2} \leq 10$ \\
\hline W0.8-3.3 & $4.4 \times 10^{4}$ & $1.3 \times 10^{4}$ & $1.0 \times 10^{4}$ \\
W1.5-2.55 & $4.8 \times 10^{4}$ & $1.5 \times 10^{4}$ & $1.2 \times 10^{4}$ \\
W0.2-2.2 & $6.0 \times 10^{3}$ & $2.5 \times 10^{3}$ & $2.0 \times 10^{3}$ \\
W359.5-3.0 & $5.6 \times 10^{3}$ & $2.8 \times 10^{3}$ & $2.3 \times 10^{3}$ \\
\hline
\end{tabular}

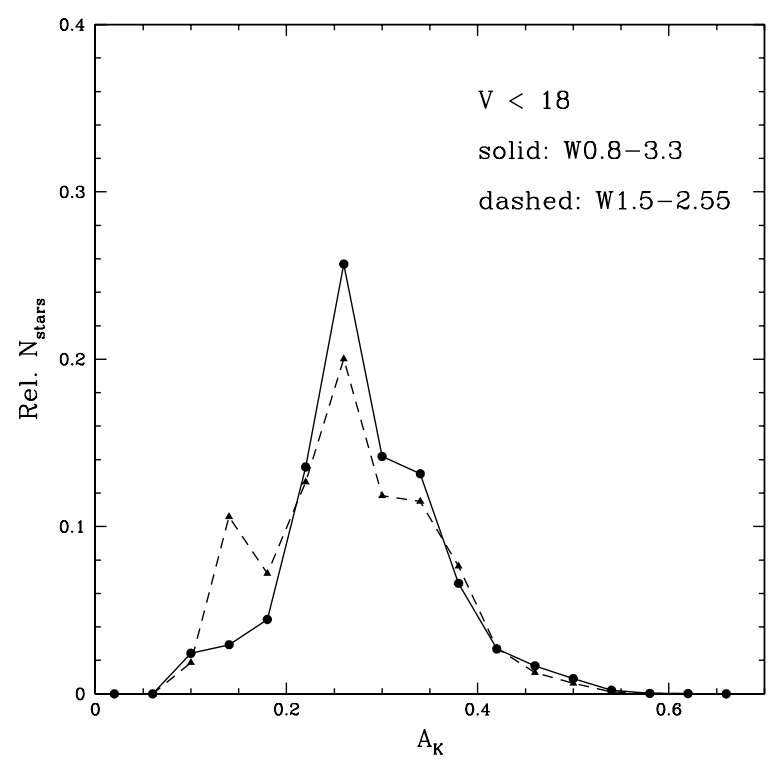

Fig. 5. Distribution of $A_{K}$ values for all stars that are in the inner bulge windows W0.8-3.3 and W1.5-2.55 and that have $V<18$, available 2MASS data, and $\chi_{\min }^{2} \leq 10$, as explained in the text.

from LNA and 3 NIR magnitudes from 2MASS; iii) minimum dispersion between its observed colours and best fitting model colours of $\chi_{\min }^{2} \leq 10$, in order to preserve the quality of the derived parameters. The evolution of the sample size as a function of these different selection criteria is shown in Table 2. Several thousand stars survive all these cut-off criteria in each region.

\subsection{Extinction}

Figures 5 and 6 show the resulting distribution of $A_{K}$ values for the 4 candidate low-extinction windows. The distributions are fairly broad. The distribution peaks at $A_{K}=0.26$ for W359.53.0 but a substantial fraction of the stars have $A_{K}$ values 0.1 higher than the peak value. For W0.2-2.2, the peak occurs at $A_{K}=0.35$. For W0.8-3.3 and W1.5-2.55 the distribution of $A_{K}$ peaks at lower values $\simeq 0.25$ and only relatively few stars are subject to $A_{K}>0.30$. In terms of optical extinction, the peak values correspond to $A_{V} \simeq 2.2$ for W1.5-2.55, W0.8-3.3 and W359.53.0, and $A_{V} \simeq 3.1$ for W0.2-2.2. This $A_{V}$ peak value for W1.52.55 is $\simeq 0.4$ mag higher than that of the SgrI window, consistent with the lower galactic latitude (Walker \& Mack 1986; Feltzing \& Gilmore 2000). As for W0.8-3.3, it is $\simeq 0.7$ mag higher than typically found in BW, again consistent with its position closer to the Galactic centre (Stanek 1996; Feltzing \& Gilmore 2000).

We therefore conclude that the 4 regions in fact have typical extinction values that are substantially lower than in their vicinity. The $A_{K}$ values found here also agree with those found by Dutra et al. (2003) based on 2MASS CMDs within square cells with $4^{\prime}$ on a side. The mean difference and dispersion 


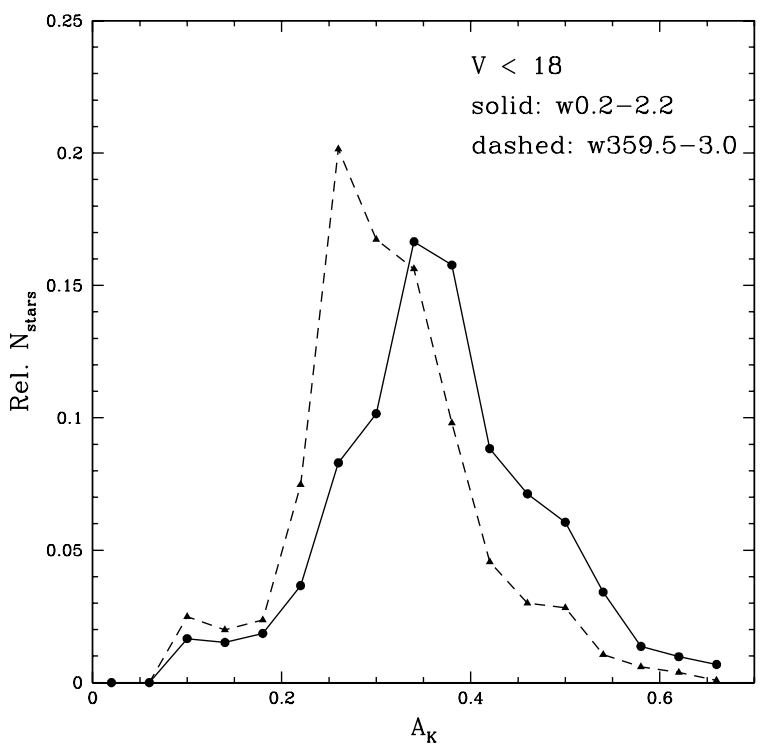

Fig. 6. Distribution of $A_{K}$ values for all stars in the inner bulge windows W0.2-2.2 and W359.5-3.0 and that have $V<18$, available 2MASS data, and $\chi_{\min }^{2} \leq 10$, as explained in the text.

between the Dutra et al. values and ours are $0.00 \pm 0.07$ for $\mathrm{W} 1.5-2.55,-0.01 \pm 0.07$ for $\mathrm{W} 0.8-3.3,-0.02 \pm 0.10$ for $\mathrm{W} 0.2-2.2$ and $-0.03 \pm 0.08$ for $\mathrm{W} 359.5-3.0$. The dispersion is very likely due to granularity in the dust distribution on smaller scales than the $4^{\prime}$ resolution of the Dutra et al. (2003) maps. Evidence of clumpiness in the inter-stellar medium, at least in the nuclear bulge (at $\leq 200$ distance from the centre), has been discussed by Launhardt et al. (2002).

The dereddened CMDs for the 4 windows are shown in Fig. 7. Notice that these CMDs are strikingly different from those shown in Fig. 4, as expected for a crowded field located in the inner Galactic bulge. Only two clear loci of stars are visible in the extinction-corrected CMDs. The disc main sequence has completely disappeared from these plots because of the $T_{\text {eff }}<5500 \mathrm{~K}$ limit of the models used in the colour fits. Also, the RC region (made up of both disc and bulge heliumburning stars) is now clearly seen and detaches itself from the bulge RGB and AGB stars, especially at brighter magnitudes. The disc RC stars in particular are seen as the dominant and nearly vertical structure at $(V-K)_{0} \simeq 2.5$ and $K_{0}<10.5$, the spread in magnitudes being due to the variable distances.

\subsection{Atmospheric parameters}

The main reason for obtaining effective temperatures and other atmospheric parameters is to use them as input values for followup spectroscopic abundance analysis, which are currently under way. The distributions of stars as a function of $T_{\text {eff }}$ is shown in Fig. 8 for the 4 regions. In all cases, the distribution is dominated by the hotter helium-burning stars in the RC, consistently with the extinction-corrected CMDs discussed in the previous section. The cut-off at the high $T_{\text {eff }}$ extreme is an artificiality caused by the limited range of the models. In fact, still hotter disc main sequence stars are present in the original sample (see Fig. 4) but have automatically been removed from the analysis because of the model limits. The secondary peak in Fig. 8 is made up of bulge RGB/AGB stars. These stars are the ones not far from the RGB/AGB tip, due to the relatively shallow photometry we have. Therefore, they are luminous and relatively cool. The more
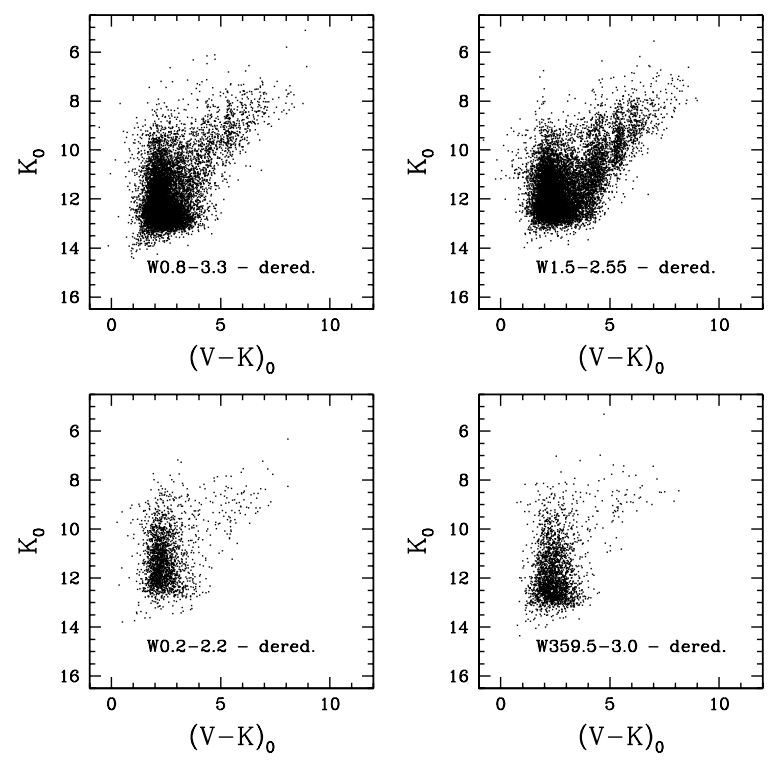

Fig. 7. $K_{0}$ vs. $(V-K)_{0} \mathrm{CMD}$ for each of the 4 bulge regions studied. The window name is indicated in each panel.
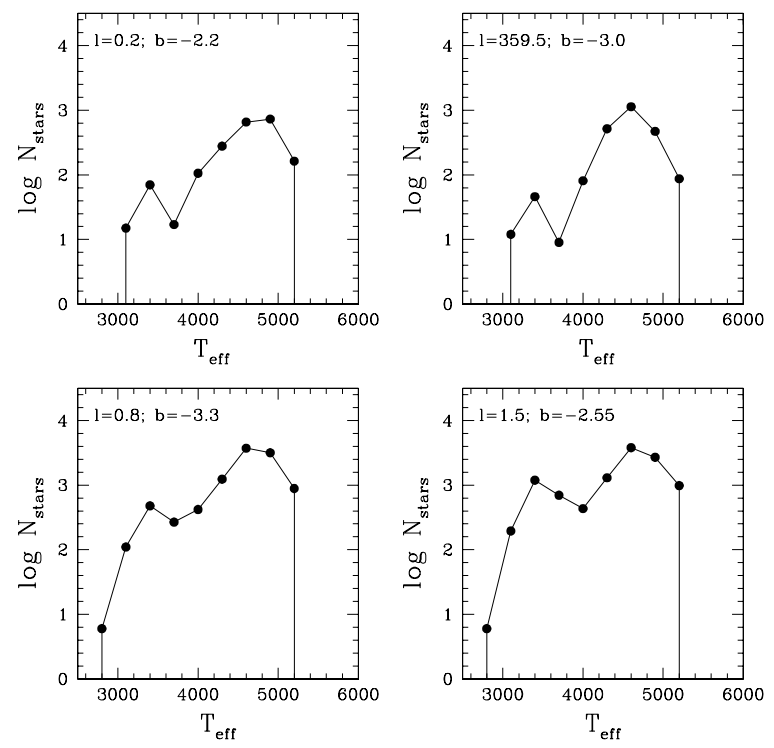

Fig. 8. Distribution of $T_{\text {eff }}$ values derived from the model vs. observed colour comparison described in Sect. 4. Each panel corresponds to one of the low-extinction windows of the inner bulge, as indicated.

numerous, hotter, and less luminous RGB stars are not favoured by our sample due to photometric incompleteness. This causes the $\operatorname{dip}$ at $T_{\text {eff }} \simeq 4000 \mathrm{~K}$.

\subsection{Metallicities}

Given the extinction-corrected photometry, based on the $A_{K}$ values obtained in Sect. 4.1, and the $K_{0}$ vs. $(V-K)_{0}$ CMD shown in Fig. 7, we are able to derive metallicities in the form of $[\mathrm{Fe} / \mathrm{H}]$ indices for the bulge upper RGB stars. We follow the same procedure as Zoccali et al. (2003), who compare the position of each RGB star in the $M_{K}$ vs. $(V-K)_{0}$ CMD with the expected position of such stars of different metallicities. The expected positions are expressed in terms of hyperbolas on the $M_{K}$ vs. $(V-K)_{0} \mathrm{CMD}$ plane whose coefficients are functions of $[\mathrm{M} / \mathrm{H}]$. The coefficients were obtained in order to make these curves fit the fiducial RGB 


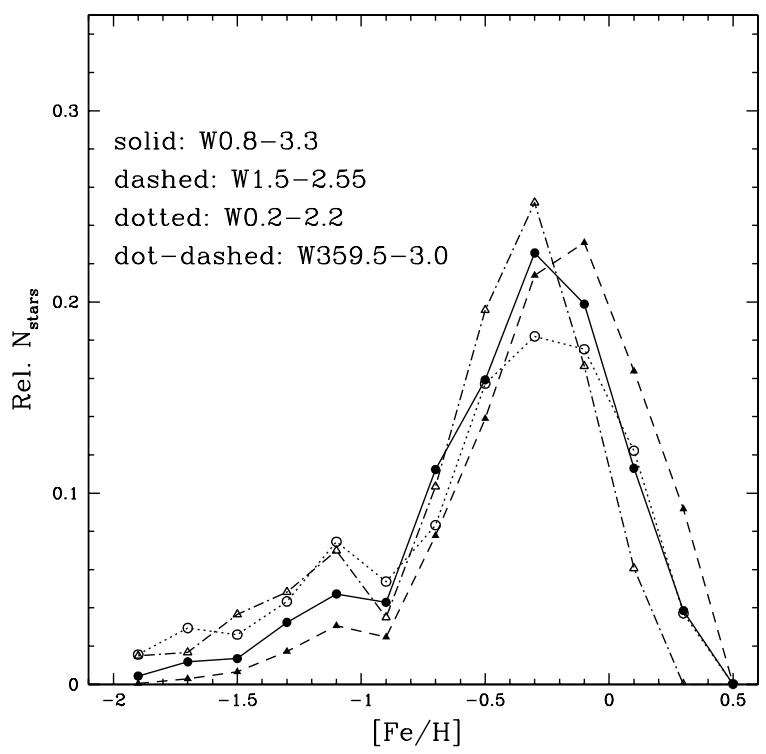

Fig. 9. Distribution of $[\mathrm{Fe} / \mathrm{H}]$ values derived with the method described in detail by Zoccali et al. (2003). The different lines and symbols correspond to the different bulge windows, as indicated.

lines of a set of well-studied Galactic globular clusters (Ferraro et al. 2000) of known metallicity. As emphasized by Zoccali et al. (2003), the sensitivity of the method is sufficient for the upper RGB alone $\left(M_{K}<-4.5\right.$ and $\left.(V-K)_{0}>2.8\right)$. We followed the same approach here and restricted ourselves only to these luminous giants, with the additional advantage that these are the stars with the highest $\mathrm{S} / \mathrm{N}$ ratios in our sample. Conversion of apparent magnitudes to absolute ones was done by assuming a distance modulus $(m-M)_{0}=14.47$ for the bulge stars. We refer to Zoccali et al. (2003) for a discussion of the expected scatter caused by variable distances relative to this value.

Thus, for each star in the upper RGB region, we found the hyperbola that passes through its CMD position and computed the $[\mathrm{M} / \mathrm{H}]$ value that yields the coefficients of this curve. The corresponding $[\mathrm{Fe} / \mathrm{H}]$ indices were computed from $[\mathrm{M} / \mathrm{H}]$ assuming $[\alpha / \mathrm{Fe}]=0.3$ for $[\mathrm{Fe} / \mathrm{H}]<-1$ and $[\alpha / \mathrm{Fe}]=0.2$ for larger metallicities. The resulting MDF is shown in Fig. 9 separately for each of our inner bulge windows. The overall shape of the MDFs is similar in all 4 cases. The MDFs are broad and peak at $[\mathrm{Fe} / \mathrm{H}] \simeq-0.25$. They drop much faster towards higher metallicities than towards lower ones. Small differences may be observed among the 4 windows: the MDFs of W0.2-2.2 and W359.5-3.0 display a small excess of stars with $[\mathrm{Fe} / \mathrm{H}]<-0.7$ relative to the other two regions. These in turn have an excess of stars with super solar values. Also, the MDF in W1.5-2.55 has a peak closer to solar metallicity at $[\mathrm{Fe} / \mathrm{H}] \simeq-0.1$. To quantify the significance of these differences, we determined median $[\mathrm{Fe} / \mathrm{H}]$ values for each distribution and ran the KolmogorovSmirnov test (KS test) among them. The median metallicities are: $[\mathrm{Fe} / \mathrm{H}]=-0.32$ for $\mathrm{W} 0.8-3.3,[\mathrm{Fe} / \mathrm{H}]=-0.22$ for $\mathrm{W} 1.5-$ $2.55,[\mathrm{Fe} / \mathrm{H}]=-0.39$ for $\mathrm{W} 0.2-2.2$ and $\mathrm{W} 359.5-3.0$. The KS test yields very weak probabilities of these MDFs being drawn from the same parent distribution, except for W0.2-2.2 and W359.53.0 , where $P=18 \%$. On the other hand, the MDF shape does not correlate with distance from the centre of the Galaxy; W0.8-3.3, for example, is the farthest from the centre among the 4 regions, but has an intermediate MDF behaviour. A weak correlation with Galactic longitude $l$ may exist, in the sense that

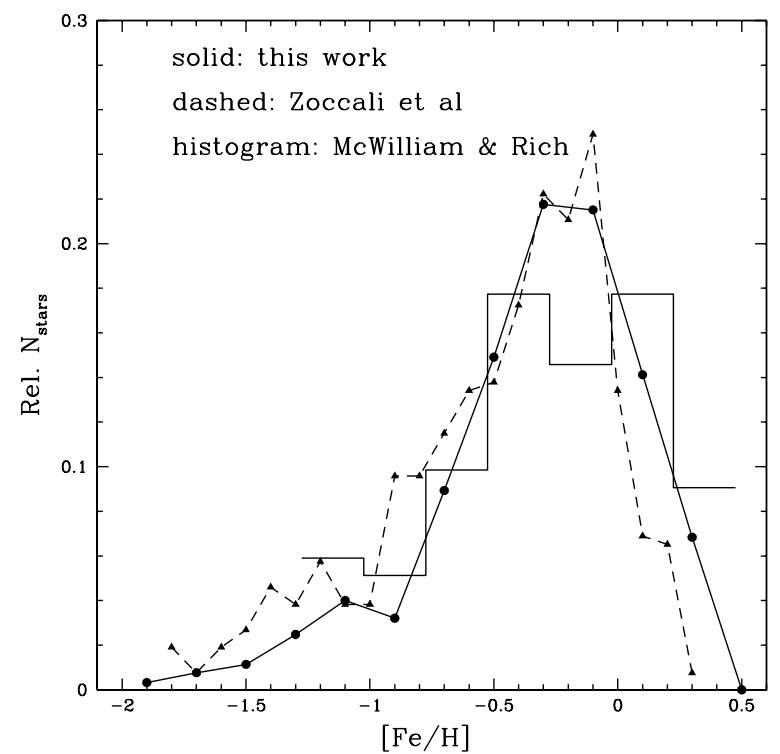

Fig. 10. Comparison between the MDF of all 4 windows studied in this work (solid line and filled circles) with the MDF from Zoccali et al. (2003) (dashed line and triangles) and the spectroscopic MDF from McWilliam \& Rich (1994) (histogram).

the median metallicity increases towards positive longitude. This might be a result of the presence of the bar (Dwek et al. 1995).

Given the relatively small differences among the different windows, we decided to put all of them together into a single MDF. Figure 10 compares this overall MDF with those obtained by other authors. In general, there is an agreement between our MDF and those of Zoccali et al. (2003) and McWilliam \& Rich (1994). The two photometrically derived MDFs have very similar peaks. The MDF by Zoccali et al. (2003) has a small excess of stars of lower metallicity $([\mathrm{Fe} / \mathrm{H}]<-0.8)$, while our composite MDF has a slight excess at supersolar values, indicating at best a very shallow gradient. Our global MDF agrees with the MDF by McWilliam \& Rich, although the latter has a slightly larger fraction of stars at lower metallicities and a broader peak.

Notice that the lower metallicity region of the photometric MDF may be affected by residual contamination of foreground disc stars belonging to the RC. This structure ends very close to the $(V-K)_{0}>2.8$ and $M_{K}<-4.5$ cut-off applied, the MDF being therefore sensitive to issues such as photometric calibration details and other systematic effects. Alternatively, the excess of lower-metallicity stars in the sample from Zoccali et al. (2003) may be caused by residual contamination by thick-disc stars in their $b=6.1^{\circ}$ field, as was observed in the fields studied by Ibata \& Gilmore (1995). Also the small excess of stars with supersolar abundances in the spectroscopic sample may reflect differences in the metallicity scales, given the different methods used to obtain the MDFs.

On the other hand, any differences in the high metallicity end among photometric samples may reflect real field-to-field variations in the MDFs. In fact, the similarity between the two photometrically derived MDFs places strong limits on any metallicity gradient between our inner fields (located $\simeq 2.4-3.5^{\circ}$ from the centre) and the one studied by Zoccali et al. (2003). The similarity between the MDFs of W0.8-3.3 and W1.5-2.55, which are respectively close to Baade's and SgrI windows, also prevents a strong gradient between them. This is consistent with the deeper photometric analysis covering smaller field areas. Feltzing \& Gilmore (2000) limit any metallicity difference at $\leq 0.2$ between 
BW and SgrI. Our results may still accommodate the mild metallicity gradient of Frogel et al. (1999), although these authors studied fields closer to the Bulge minor axis. In the next section we discuss the implications of our results in combination with previous studies.

\section{Summary and conclusions}

We have presented $B V R I$ photometry for over $10^{5}$ stars with $V \leq 18$ located in 4 regions within the central $3.5^{\circ}$ of the Galaxy. About $1 / 3$ of these stars have been astrometrically matched to the 2MASS database, yielding a large sample of stars with available optical and NIR photometry. The several observed colour indices obtained were compared to model colours in order to infer extinction and effective temperature for each star individually. The resulting distribution of $A_{K}$ values in each direction peaks at similar values as those from the Dutra et al. (2003) extinction maps, which were obtained in $4^{\prime} \times 4^{\prime}$ cells. In particular, we confirm the results by Dutra et al. (2002) that these regions are of relatively low extinction when compared to nearby fields. The comparatively low extinction allows a large contribution of inner Galactic bulge stars to the observed star counts, showing a clear excess to the expected counts from the disc. The bulge $\mathrm{RGB}, \mathrm{AGB}$, and red clump are all clearly visible in our data CMDs, especially when they are corrected for extinction.

The MDF of the bulge RGB stars was obtained from the $K_{0}$ vs. $(V-K)_{0}$ CMD following the same approach as described by Zoccali et al. (2003). When considered individually, the MDFs of the 4 low extinction regions are similar, peaking at $[\mathrm{Fe} / \mathrm{H}] \simeq-0.25$ and having a much sharper drop off at the high-metallicity end than at the low-metallicity end. However, small but statistically significant differences are found among the different regions, as the different median metallicities and the results of the KS test attest. The global MDF from all 4 fields was compared to those from Zoccali et al. (2003) and from the spectroscopic sample of McWilliam \& Rich (1994). Our MDF is very similar to that of Zoccali et al. (2003), especially for $[\mathrm{Fe} / \mathrm{H}]>-0.5$. At lower metallicities the latter MDF shows an excess of stars relative to ours, but in a range of $[\mathrm{Fe} / \mathrm{H}]$ where contamination from disc RC stars may be an issue. The MDF derived here also agrees with that of McWilliam \& Rich (1994), apart from the fact that the latter seems to be a broader distribution. The similarities among the MDFs advocates against any significant abundance variations or an abundance gradient in the bulge within the $2.2^{\circ} \leq \theta_{\mathrm{c}} \leq 6.0^{\circ}$ range, where $\theta_{\mathrm{c}}$ is the angular distance from the Galactic centre. In physical scales, this corresponds to the range from 300 to $900 \mathrm{pc}$ (assuming $R_{0}=8.5 \mathrm{kpc}$ ). Therefore, the inner bulge may have formed in a process in which energy dissipation was not significant. A hierarchical process involving accretion of different stellar fragments is therefore favoured.

The broad range in metallicities of the inner bulge stars may then, at least partially, reflect the enrichment history of the fragments prior to being accreted into the central regions of the Galaxy. Larger fragments may have retained more of their enriched gas, allowing star formation to proceed towards higher metallicities than lower mass fragments. On the other hand, the physical range probed here is perhaps small enough, and the stellar density high enough that dynamical effects in these regions may have erased or strongly smoothed any original gradient that the early bulge may have developed. Since a metallicity gradient has been observed within the central $2 \mathrm{kpc}$ (Minniti et al. 1995) and beyond (Frogel et al. 1999), it is important to establish the transition range where the gradient starts manifesting itself, something that requires analysis of bulge regions at $\simeq 6-10^{\circ}$ from the centre.

We have obtained mid-resolution CTIO/Hydra and Gemini/GMOS spectra for about 200 stars towards these low-extinction regions in the inner bulge. The extinction and effective temperature values obtained in this work are being used along with the spectra in order to obtain spectroscopic metallicities and estimates of $[\alpha / \mathrm{Fe}]$. These data will allow us to compare the photometric and spectroscopic metallicity scales and also provide assessment of the timescales of star formation in these central regions. Finally, it is worth mentioning that the 4 windows studied here are located at $-3.3 \leq b \leq-2.15$. As the large-scale bar has a scale height of $\simeq 0.8 \mathrm{kpc}$, these windows are placed within or close to the zone of influence of the bar. There is also evidence of an inner bar-like structure (Nishiyama et al. 2005). Thus we have guaranteed time at LNA for new and deeper observations of these and other comparison fields to study the bar vertical structure.

Acknowledgements. BXS and SCJ acknowledge the support of Conselho Nacional de Desenvolvimento Científico e Tecnológico ( $\mathrm{CNPq}$ ) in the form of fellowships and material resources. BXS also thanks the Fundação de Amparo à Pesquisa do Rio Grande do Sul (FAPERGS) for travel grants related to this work. GFPM acknowledges financial support from FAPERJ under grant E-26/170.687/2004, from CNPq/Brazil under grant 552331/01-5, and from the MEGALIT/Instituto do Milênio program. The authors are grateful to the referee, D. Valls-Gabaud, for his very interesting and useful comments. The staff at the Laboratório Nacional de Astrofísica were very efficient and provided all the logistics and instrumental support needed to carry out the observations.

\section{References}

Anthony-Twarog, B., Mukherjee, K., Cladwell, N., \& Twarog, B. 1988, AJ, 95, 1453

Baade, W. 1951, Publ. Obs. Univ. Michigan, 10, 7

Babusiaux, C., \& Gilmore, G. 2005, MNRAS, 358, 1309

Bessel, M., Castelli, F., \& Plez, B. 1998, A\&A, 333, 231

Catchpole, R., Whitelock, P., \& Glass, I. 1990, MNRAS, 247, 479

Dwek, E., Arendt, R. G., Hauser, M. G., et al. 1995, ApJ, 445, 716

Dutra, C., Santiago, B., \& Bica, E. 2002, A\&A, 381, 219

Dutra, C., Santiago, B., Bica, E., \& Barbuy, B. 2003, MNRAS, 338, 253

Epchtein, N., de Batz, B., Capoani, L., et al. 1997, The Messenger, 87, 27

Feltzing, S., \& Gilmore, G. 2000, A\&A, 355, 949

Ferraro, F., Montegriffo, P., Origlia, L., \& Fusi Pecci, F. 2000, AJ, 119, 1282

Frogel, J., Tiede, G., \& Kuchinski, L. 1999, AJ, 117, 2296

Ibata, R., \& Gilmore, G. 1995, MNRAS, 275, 605

Kim, S., \& Morris, M. 2001, ApJ, 554, 1059

Kjeldesen, H., \& Frandsen, S. 1991, A\&AS, 87, 119

Launhardt, R., Zylka, R., \& Mezger, P. 2002, A\&A, 384, 112

Lépine, J., \& Leroy, P. 2000, MNRAS, 313, 263

McWilliam, A., \& Rich, R. M. 1994, ApJS, 91, 749

Minniti, D., Olszewski, E., Liebert, J., et al. 1995, MNRAS, 277, 1293

Morris, M., \& Serabyn, E. 1996, ARA\&A, 34, 645

Nishiyama, S., Nagata, T., Baba, D., et al. 2005, ApJL, 621, 105

Piatti, A., Claria, J., \& Bica, E. 1998, ApJS, 116, 263

Picaud, S., \& Robin, A. C. 2004, A\&A, 428, 891

Plez, B., Brett, J., \& Nordlund, A. 1992, A\&A, 256, 551

Prisinzano, L., Micela, G., Sciortino, S., \& Favata, F. 2004, A\&A, 417, 945

Ramírez, I., \& Meléndez, J. 2005, ApJ, 626, 465

Ramírez, S., Stephens, A., Frogel, J., \& DePoy, D. 2000, AJ, 120, 833

Sadler, E. M., Rich, M., \& Terndrup, D. M. 1996, AJ, 112, 171

Schultheis, M., Ganesh, S., Simon, G., et al. 1999, A\&A, 349, L69

Serabyn, E., \& Morris, M. 1996, Nature, 382, 602

Skrutskie, M., et al. 1997, in The Impact of Large Scale Near-IR Sky Surveys, ed. Garzon et al. (Dordrecht: Kluwer), 25

Stanek, K. Z. 1996, AJ, 460, L37

Stanek, K. 1998, Using the DIRBE/IRAS all-sky reddening map to select lowreddening windows near the Galactic Plane [arXiv: astro-ph/9802307]

Tiede, G., Frogel, J., \& Terndrup, D. 1995, AJ, 110, 2788

VandenBerg, D., \& Clem, J. 2003, AJ, 126, 778 (VC03)

Walker, A., \& Mack, P. 1986, MNRAS, 220, 69

Zoccali, M., Renzini, A., Ortolani, S., et al. 2003, A\&A, 399, 931 


\section{Online Material}


B. X. Santiago et al.: Multiband photometry in the inner bulge, Online Material $p 2$

Table A.1. Standard stars.

\begin{tabular}{lcccccc}
\hline \hline Field: Star & $\begin{array}{c}\text { RA(1985) } \\
(\mathrm{h}: \mathrm{m}: \mathrm{s})\end{array}$ & $\begin{array}{c}\text { Dec(1985) } \\
\left({ }^{\circ}:^{\prime}:^{\prime \prime}\right)\end{array}$ & $V$ & $B-V$ & $V-R V-I$ \\
\hline SA105: 205 & $13: 35: 07$ & $-00: 53: 16$ & 8.798 & 1.363 & 0.744 & 1.419 \\
SA105: 214 & $13: 35: 30$ & $-00: 51: 10$ & 7.062 & 0.528 & 0.313 & 0.624 \\
SA109: 231 & $17: 44: 34$ & $-00: 25: 31$ & 9.331 & 1.462 & 0.786 & 1.492 \\
SA109: 537 & $17: 44: 56$ & $-00: 21: 15$ & 10.358 & 0.608 & 0.377 & 0.769 \\
SA110: 441 & $18: 42: 48$ & $+00: 18: 44$ & 11.124 & 0.553 & 0.325 & 0.663 \\
SA110: 450 & $18: 43: 06$ & $+00: 22: 01$ & 11.585 & 0.950 & 0.552 & 1.181 \\
SA111: 717 & $19: 36: 09$ & $+00: 05: 29$ & 8.529 & 0.425 & 0.236 & 0.483 \\
SA111: 773 & $19: 36: 30$ & $+00: 08: 56$ & 8.963 & 0.206 & 0.118 & 0.261 \\
SA111: 775 & $19: 36: 30$ & $+00: 10: 02$ & 10.747 & 1.738 & 0.965 & 1.864 \\
SA112: 595 & $20: 40: 32$ & $+00: 13: 14$ & 11.349 & 1.598 & 0.899 & 1.800 \\
SA112: 636 & $20: 40: 49$ & $+00: 13: 18$ & 9.853 & 0.688 & 0.402 & 0.799 \\
SA114: 750 & $22: 40: 59$ & $+01: 07: 54$ & 11.913 & -0.038 & 0.030 & 0.013 \\
SA114: 755 & $22: 41: 22$ & $+01: 12: 06$ & 10.908 & 0.571 & 0.311 & 0.621 \\
SA114: 670 & $22: 41: 24$ & $+01: 05: 34$ & 11.106 & 1.203 & 0.645 & 1.224 \\
\hline
\end{tabular}

\section{Appendix A:}

\section{A.1. Photometric calibration}

A total of 15 observations of 5 SA fields were made on the night of June 26, 2003. More than one exposure with each filter was taken in each observation. The total number of exposures of standard stars on this night was 223, typically more than 50 with each filter. On the following night, June 27th, 6 SA fields were observed interchangeably, totalling 16 observations and 214 exposures in all 4 filters. The list of SA fields and standard stars used is given in Table A.1, along with their positions, magnitudes, and colours. The range in observing times and airmasses was the same as for the bulge fields and clusters. Exposure times for the calibration images varied from $1 \mathrm{~s}$ to $40 \mathrm{~s}$, depending on the apparent magnitude of the standard stars. As the magnitudes vary considerably, even within a single SA field, several individual magnitude measurements were discarded due to saturation or to low $\mathrm{S} / \mathrm{N}$. The typical $\mathrm{S} / \mathrm{N}$ for the calibrated stars is comparable to those of $V \simeq 14$ in the bulge fields.

Instrumental magnitudes, measured in 2 pixel apertures were transformed to the Johnson-Cousins system by means of the following transformation equations:

$$
\begin{aligned}
& B=B_{\text {inst }}+a_{b}(B-V)+b_{b} X+c_{b} \\
& V=V_{\text {inst }}+a_{v}(V-I)+b_{v} X+c_{v} \\
& R=R_{\text {inst }}+a_{r}(V-R)+b_{r} X+c_{r} \\
& I=I_{\text {inst }}+a_{i}(V-I)+b_{i} X+c_{i} .
\end{aligned}
$$

In the equations above, the left-hand side corresponds to the calibrated magnitudes, $X$ is the airmass, and the index "inst" corresponds to the instrumental magnitudes. The transformations above were applied iteratively until the calibrated magnitudes and their associated colours converged within $0.01 \mathrm{mag}$. The resulting calibrated magnitudes were tested for alternative transformations involving other colour indexes, with and without a quadratic colour term. The calibrated magnitudes were robust to use of these alternative equations. Inclusion of a quadratic term did not improve the residuals. Notice that the aperture correction from 2 pix to infinity is automatically absorbed by the zero-point term in the calibration equations.

The values of the calibration coefficients are given in Table A.2 for the two nights. Notice that the airmass terms in general increase towards shorter wavelengths, as expected.
Table A.2. Calibration equations.

\begin{tabular}{ccccc}
\hline \hline Night & equation & colour term & X term & ZP \\
\hline $06 / 26$ & $I(V-I)$ & +0.022 & -0.080 & -0.133 \\
$06 / 26$ & $R(V-R)$ & -0.077 & -0.138 & +0.353 \\
$06 / 26$ & $V(V-I)$ & -0.038 & -0.362 & +0.383 \\
$06 / 26$ & $B(B-V)$ & +0.085 & -0.390 & -0.102 \\
$06 / 27$ & $I(V-I)$ & +0.037 & -0.037 & -0.221 \\
$06 / 27$ & $R(V-R)$ & -0.041 & -0.252 & +0.452 \\
$06 / 27$ & $V(V-I)$ & -0.058 & -0.242 & +0.283 \\
$06 / 27$ & $B(B-V)$ & +0.140 & -0.326 & -0.217 \\
\hline
\end{tabular}

\section{A.2. Astrometry}

Chip $(x, y)$ positions were converted into equatorial coordinates through a process that involved several steps. First, a search for Guide Star Catalogue 1.2 (GSC) stars within a few selected LNA fields was made with the Vizier (http://vizier.u-strasbg.fr/) database. The onsky VO plot of GSC stars was then compared to the positions of the 100 brightest stars in these selected LNA fields, and visual matches were identified. Typically, from 30 to 40 matches were found in a single field. A CCD pixel-to-coordinate transformation was then carried out for these matched stars. The transformation equation was of the kind

$$
\begin{aligned}
\mathrm{RA}= & \mathrm{RA}_{0}+A+B\left(X-X_{\mathrm{c}}\right)+C\left(Y-Y_{\mathrm{c}}\right)+D\left(X-X_{\mathrm{c}}\right)^{2} \\
& +E\left(Y-Y_{\mathrm{c}}\right)^{2}+F\left(X-X_{\mathrm{c}}\right)\left(Y-Y_{\mathrm{c}}\right) \\
\operatorname{Dec}= & \operatorname{Dec}_{0}+P+Q\left(X-X_{\mathrm{c}}\right)+R\left(Y-Y_{\mathrm{c}}\right)+S\left(X-X_{\mathrm{c}}\right)^{2} \\
& +T\left(Y-Y_{\mathrm{c}}\right)^{2}+U\left(X-X_{\mathrm{c}}\right)\left(Y-Y_{\mathrm{c}}\right)
\end{aligned}
$$

where $\left(X_{\mathrm{c}}, Y_{\mathrm{c}}=512,512\right)$ correspond to the mid-CCD pixel position, $\left(\mathrm{RA}_{0}, \mathrm{Dec}_{0}\right)$ correspond to the nominal central equatorial coordinates of the LNA field (J2000.0). The coefficients $(\mathrm{A}, \mathrm{B}, \mathrm{C}, \mathrm{D}, \mathrm{E}, \mathrm{F})$ were then varied so as to minimise the dispersion between the fitted RA values and the GSC RA ones. Likewise for declination, the best set of (P, Q, R, S, T, U) parameters was again found so as minimise dispersion. The astrometric solutions found for these selected fields was very stable: all quadratic coefficients (D, E, F, S, T, U) proved to be negligible. As the LNA direct camera is oriented so that north-south direction runs along the $\mathrm{X}$ direction, the terms $\mathrm{B}$ and $\mathrm{R}$ were small (varying from 0.0023 to 0.0032 ). As for $\mathrm{C}$ and $\mathrm{Q}$, their values were also very stable and reflected the pixel scale on the CCD. The largest variations were seen in the field centre corrections, $A$ and $P$.

Given the observed stability in the astrometry, this type of transformation was applied to all fields, allowing for large variations only in field centre, and smaller variations in the linear parameters (with the quadratic ones set to zero). The transformation parameters were varied until the transformed (RA, Dec) LNA positions were visually very well-matched to the observed GSC ones on the sky. An excellent match for the GSC stars in each LNA was always possible in this way, with relatively small variation in the parameters. As our final goal was to cross our optical sample from LNA with the 2MASS NIR data, a third step in astrometric solutions was applied to all fields: zero-point and linear astrometric coefficients were allowed to vary until the largest number of matches between the brightest stars of both samples was achieved. Again, the difference between the bestfitting 2MASS parameters and the best-fitting GSC ones proved to be very small for all fields, attesting the stability of the astrometric transformations. 


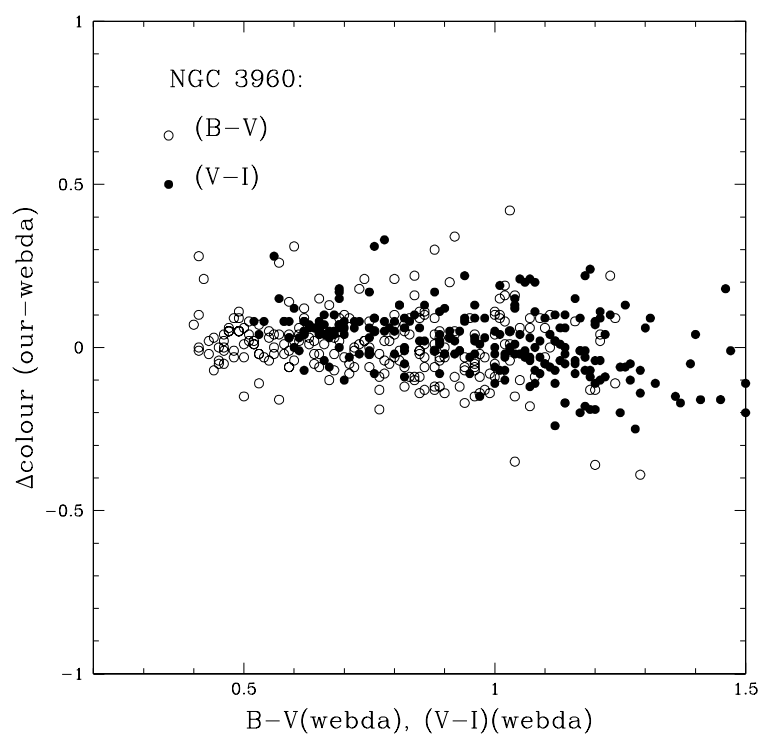

Fig. A.1. Colour differences between this work and that of Prisinzano et al. (2004) for NGC 3960 stars. Both $(B-V)$ and $(V-I)$ colours are shown, as indicated.

\section{A.3. Cluster data}

The photometry was tested against CCD data found in the literature for two open clusters: NGC 3960 and IC4651. They were observed at LNA on the nights of 26/06 and 27/06, respectively. Previous data for these clusters were found in the Webda database. NGC 3960 was observed by Prisinzano et al. (2004). The CCD photometry for IC 4651 is found in Anthony-Twarog et al. (1988), Piatti et al. (1998) and Kjeldsen \& Frandsen (1991).

Figure A.1 shows the $(B-V)$ and $(V-I)$ colour differences for one of the clusters, NGC 3960. There is no systematic bias in the colours as compared to previous CCD measurements. There may be a very slight trend in $(V-I)$ in the sense that our colours are a bit bluer than those of Prisinzano et al. (2004) for the red stars. The standard deviation in the distribution of all colour differences is $\sigma_{B-V}=0.10$ and $\sigma_{V-I}=0.10$. Similar results hold for IC 4651. 